\title{
Cystine Crystal Measurement
}

National Cancer Institute

\section{Source}

National Cancer Institute. Cystine Crystal Measurement. NCI Thesaurus. Code C74674.

The determination of the amount of cystine crystals present in a sample. 